\title{
Las ecuaciones diferenciales lineales de Segundo Orden como modelos matemáticos
}

\section{The lineal differential equations of Second Order as mathematic models}

\author{
Equações diferenciais lineares de segunda ordem como modelos matemáticos \\ Rosa Virginia Hernández, Luis Fernando Mariño, Mauricio Penagos. \\ Magister"en Educación Matemáticas, Docente, Universidad Francisco de Paula Santander; Cúcuta, Colombia $(1)$ \\ Magister en Educación matemáticas, Docente, Universidad Francisco de Paula Santander; Cúcuta, Colombia (1) \\ Maestro en Ciencias en Física, Docente, Universidad Surcolombiana Neiva, Colombia (1)
}

Forma de citar: Hernández, R., Mariño, F., Penagos, M. (2016). Las ecuaciones diferenciales lineales de Segundo Orden como modelos matemáticos. Encuentro Internacional en Educación Matemática Segunda Versión ISSN 2539-1885. La Educación Matematica como Herramienta en el Desempeño Profesional Docente. Cúcuta, Colombia. 54 - 60.

Resumen: La resolución de problemas y modelación matemáticas son áreas críticas en la enseñanza y aprendizaje de la matemática. Allí se deben poner en juego, conceptos, habilidades y procedimientos provenientes de la experiencia matemática en cursos anteriores. La mayoría de los estudiantes tienen dificultades para llegar a entender el lenguaje de las matemáticas; relacionadas con el conocimiento inadecuado del lenguaje especializado que incluye palabras técnicas, no técnicas, y notación simbólica, específicamente en la formulación de modelos matemáticos. El propósito del estudio estuvo centrado en analizar los resultados sobre el conocimiento semántico que un grupo de estudiantes de la Facultad de Ingeniería de la Universidad Francisco de Paula Santander evidencia en la representación de ecuaciones diferenciales lineales de segundo orden como modelos matemáticos. Los fundamentos teóricos de que dieron soporte a la investigación fueron: La teoría de dos etapas propuesta por (Mayer, 1986), el ciclo de modelación bajo la perspectiva cognitiva de (Ferri, 2006) y las representaciones externas de (Goldin \& Kaput, 1996). El trabajo fue cuantitativo de tipo exploratorio y descriptivo. La investigación se fundamentó en la teoría de dos etapas propuesta por Mayer R para la resolución de problemas matemáticos, el ciclo de modelación según Ferri y la teoría de las representaciones de Goldin y Kaput. Para recolectar la información, se diseñó y aplicó un cuestionario de 17 reactivos con respuestas cerradas y abiertas. Los hallazgos muestran que cada participante hace su propia representación interna y externa a conceptos como: sistema masa-resorte, peso, masa, punto de equilibrio, Ley de Hooke, fuerzo amortiguadora, fuerza externa, Ley de Newton inmersos en la situación mediante un problema de palabra. Es necesario realizar trabajos a profundidad sobre el conocimiento con el propósito de buscar explicaciones y contribuir en la enseñanza y aprendizaje hacia la resolución de problemas matemáticos.

Palabras clave Ciclo de modelación, modelación matemática, problemas matemáticos, representaciones externas.

Abstract: The resolution of problems and mathematics modeling are critic areas in learning and teaching of mathematics. Is there where it must to put on game concepts, skills and procedures originating from the mathematic experience in previous courses. Most of the students have difficulties to understand the mathematic language, related with the inadequate knowledge of specialized language that includes technique words, non-technique words and symbolic notations, specifically in the formulation of mathematic models. The purpose of this research was focused to analyze the results about the semantic knowledge that a group of students of Engineering Faculty of Francisco of Paula Santander University evidence in the representation of lineal differential equations of second order as mathematic models. The theory fundaments that gave support the research was: The theory of two phases by (Mayer, 1986), the modeling cycle under the cognitive perspective of (Ferry, 2006) and the extern representations of (Goldin \& Kaput, 1996). The project was quantitative of exploratory and descriptive type. The research was based in the theory of two phases purposed by Mayer R for the resolution of mathematic problems, the modeling cycle according Ferry and the Representations theory of Goldin and Kaput. To recollect the information it was designed and applied a questionary of 17 reactive with opened and closed answers. The discoveries showed that each participant does its own intern an extern representation to concepts as: spring-mass system, weight, mass, balance point, Hooke's Law, buffering strong, extern strong, Newtown's Law immersed in a situation through a problem of word. It is necessary to execute deep jobs about the knowledge with the purpose of to look for explanations and aid in teaching and learning through the resolution of mathematic problems.

Key words Modeling cycle, Mathematic Modeling, Mathematic problems, extern representations.

* Autor para correspondencia rosavirginia@ufps.edu.co

2590-9215@ 2017 Universidad Francisco de Paula Santander. Este es un artículo bajo la licencia CCBY 
Resumo: Resolução de problemas e modelagem matemática são áreas críticas no ensino e aprendizagem da matemática. Deve ser colocado em jogo, conceitos, habilidades e procedimentos a partir da experiência matemática em cursos anteriores. A maioria dos alunos tem dificuldade em entender a linguagem da matemática; relacionado ao conhecimento inadequado de linguagem especializada que inclui palavras técnicas, não técnicas, e notação simbólica, especificamente na formulação de modelos matemáticos. O objetivo do estudo incidiu sobre a análise dos resultados sobre o conhecimento semântico que um grupo de estudantes da Faculdade de Engenharia da evidência Universidade Francisco de Paula Santander na representação de equações diferenciais lineares de segunda ordem como modelos matemáticos. Os fundamentos teóricos que deram apoio à pesquisa foram: A teoria de dois estágios proposto por (Mayer, 1986), o ciclo de modelagem sob a perspectiva cognitiva (Ferri, 2006) e representações externas (Goldin \& Kaput de 1996 ). O trabalho foi quantitativo de tipo exploratório e descritivo. A pesquisa foi baseada na teoria de dois estágios proposta por Mayer R para resolver problemas matemáticos, o ciclo de modelagem de acordo com Ferri e a teoria das representações de Goldin e Kaput. Para coletar as informações, foi elaborado e aplicado um questionário de 17 itens com respostas fechadas e abertas. Os resultados mostram que cada participante faz sua própria representação interna e externa para conceitos tais como o sistema massa-mola, peso, massa, equilíbrio, a Lei de Hooke, força de amortecimento mim, força externa, Act imerso Newton na situação através de um problema da palavra. É necessário realizar um trabalho aprofundado sobre conhecimento com o objetivo de buscar explicações e contribuir para o ensino e a aprendizagem para a solução de problemas matemáticos.

Palavras-chave: Ciclo de modelagem, modelagem matemática, problemas matemáticos, representações externas.

\section{Introducción}

La ingeniería, como en otros programas, necesita de las matemáticas para lograr sus propios fines ya que estas le permiten dar solución a los problemas planteados que provienen de la industria o del diario vivir, (Gutiérrez, 2014). Los programas académicos para la formación de ingenieros se caracterizan por tener un amplio componente de matemáticas especialmente en el ciclo básico; sin embargo, "la modelación matemática es uno de los temas que aparece oculto de las carreras universitarias"; es decir, no existe ninguna asignatura de la ingeniería que se dedique a la enseñanza aprendizaje de los estudiantes para modelar independientemente de las áreas que se articulan (Gallardo, s.f).

Las Ecuaciones diferenciales es una de las asignaturas que más aporta en la formación del ingeniero y también quizá es una de las que más dificultad de aprendizaje presenta; especialmente, cuando un estudiante se enfrenta a problemas de modelado debe poner en juego, conceptos, habilidades y procedimientos provenientes del cálculo diferencial, integral, álgebra lineal, estadística e incluso de lo visto en el nivel educativo precedente. Según (Blomhoj , 2008), la modelación matemática es el proceso de traducción entre el mundo real y las matemáticas en ambas direcciones. Para (Camarena , 2004), la modelación matemática es que el modelo que se concibe como el proceso cognitivo que se tiene que llevar a cabo para llegar a la construcción del modelo matemático de un problema u objeto del área de contexto" (p.20). Según (Mayer , 1986), para resolver un problema se debe pasar por las etapas: Traducción y solución para la primera se requiere conocer el significado en el lenguaje natural de cada una de las palabras que aparecen en el enunciado (conocimiento lingüístico) y conocimiento de los hechos acerca del mundo real (conocimiento semántico), para la segunda conocimiento operativo y estratégico; estos procesos cognitivos requieren y destacan la importancia del tránsito que tiene que hacer el resolutor del lenguaje natural al lenguaje matemático.
En esta fase se presentan grandes dificultades que pueden originar malentendidos, que se producen cuando el solucionador de problemas construye un modelo mental de la situación problemática que entra en conflicto con la información en el enunciado del problema (Mayer, Lewis, \& Hegarty, 1992 ). La representación de situaciones del mundo real o problemas de palabra es una etapa crucial en la formulación de modelos matemáticos. Un alto porcentaje de los estudiantes no llega a entender el lenguaje matemático especializado que incluye palabras técnicas, no técnicas y su respectiva notación de la simbología, (Sabbagh, 2008, concluye sobre uno de los aspectos del fracaso hacia la enseñanza aprendizaje de las matemáticas en la resolución de problemas redactados o verbales es la incapacidad para suprimir la información irrelevante del mismo y esto debe a la carencia de control inhibitorio cognitivo.

Según (Calle Palomeque, 2013), el problema semántico del lenguaje matemático es muy complejo, debido a la diversidad de registros semióticos utilizados en la actividad matemática, como: "el uso del lenguaje ordinario oral y escrito, símbolos específicos, representaciones graficas de objetos materiales y un sinnúmero de signos" (p.22). Mientras que para (Blum \& Ferri, 2009), las posibles causas de las dificultades en los procesos de modelación radica en las demandas cognitivas de las tareas, puesto que se encuentran ligadas a otras competencias como la lectura, comunicación, el diseño y la aplicación de estrategias para la resolución de problemas" (p.46). Investigadores como (Haghverdi, Semnani, \& Seifi, 2012), (Calle Palomeque, 2013), ((Bassanezi \& Biembengut, 1997), coinciden en que los errores de los alumnos en la resolución de problemas son resultado de la falta de conocimiento lingüístico, semántico, estructural y comunicacional, el empleo del proceso semántico y del lenguaje matemático es deficiente, no interpretan ni relacionan signos ya aprendidos con los nuevos, desconociendo el proceso semántico del significado de las palabras. 
Para ((Berdugo, 2004), la dificultad reside en el emparejamiento entre la comprensión del texto, la situación constituida en el texto y la representación matemática. Según (Wright, 2014), la mayor dificultad se encuentra en la fase de traducción del lenguaje humano al simbolismo matemático. Estos investigadores coinciden en muchos aspectos, pero el relevante para la investigación es el primer paso: entender el problema y formular el modelo. Según (Polya, 2005), esto requiere que el resolutor entienda el significado del problema, si puede replantear o escribir el problema utilizando sus propias palabras, identificar datos conocidos y variables. Entre tanto para (Shonenfeld, 1980), lo primero es la categoría de recursos entendida como conocimientos previos que posee el individuo, la forma en que el profesor accede a los conceptos que tiene el estudiante, circunstancias estereotípicas que provocan respuestas como un simple procedimiento y los recursos defectuosos (algún conocimiento mal aprendido, así sea una fórmula).

La enseñanza y aprendizaje de las matemáticas son un "proceso intencionado de apropiación del conocimiento matemático, que se inicia con la reflexión, comprensión, construcción y evaluación de las acciones didácticas que propician la adquisición y desarrollo de habilidades y actitudes para un adecuado desempeño en la sociedad" (p. 255), en tanto (Camarena , 2012), menciona que "la matemáticas en ingeniería es un lenguaje, puesto que casi todo lo que se dice en ingeniería se puede representar en lenguaje matemático" (p. 5), y que la modelación es uno de los temas que aparece en el currículo oculto, pero los profesores de matemáticas asumen que esto compete sólo a profesores de cursos propios de ingeniería; en contraste (Santos, 2008), manifiesta que deben seleccionarse apropiadamente problemas o actividades que promuevan el desarrollo del pensamiento matemático en los estudiantes, que le ayuden a construir una matemática para la vida, como herramienta de apoyo y disciplina formativa con una función específica en el nivel universitario donde los conocimientos deben ser integrados.

Además, la matemática es una de las ciencias básicas fundamentales en ingeniería, en cualquiera de sus especialidades; según (Cruz , 2010), hay dos razones fundamentales: por una parte se encuentra el conjunto de competencias intelectuales que se desarrollan al estudiar contenidos matemáticos (observación, análisis, comparación, síntesis, etc. y b) el hecho de constituir un poderoso lenguaje de comunicación de conocimientos de otras ramas que se usan en la formulación de situaciones ligadas a los llamados problemas de ingeniería (física, química, mecánica, electricidad, etc.), (p. 39). Por la trascendencia que tiene esta etapa en los procesos de modelación, la investigación se centra en éste elemento fundamental, analizando la estructura del lenguaje natural que requiere ser matematizado, y en el marco del planteamiento de (Mayer, 1986), acerca de los estadios para resolver problemas, se planteó el siguiente objetivo para orientar la investigación:
Determinar el tipo de significado que se establece en el conocimiento semántico cuando formulan ecuaciones diferenciales lineales de segundo orden como modelos matemáticos.

Para ((Berdugo, 2004), la dificultad reside en el emparejamiento entre la comprensión del texto, la situación constituida en el texto y la representación matemática. Según (Wright, 2014), la mayor dificultad se encuentra en la fase de traducción del lenguaje humano al simbolismo matemático. Estos investigadores coinciden en muchos aspectos, pero el relevante para la investigación es el primer paso: entender el problema y formular el modelo. Según (Polya, 2005 ), esto requiere que el resolutor entienda el significado del problema, si puede replantear o escribir el problema utilizando sus propias palabras, identificar datos conocidos y variables.

Entre tanto para (Shonenfeld, 1980), lo primero es la categoría de recursos entendida como conocimientos previos que posee el individuo, la forma en que el profesor accede a los conceptos que tiene el estudiante, circunstancias estereotípicas que provocan respuestas como un simple procedimiento y los recursos defectuosos (algún conocimiento mal aprendido, así sea una fórmula).

La enseñanza y aprendizaje de las matemáticas son un "proceso intencionado de apropiación del conocimiento matemático, que se inicia con la reflexión, comprensión, construcción y evaluación de las acciones didácticas que propician la adquisición y desarrollo de habilidades y actitudes para un adecuado desempeño en la sociedad" (p. 255), en tanto (Camarena , 2012), menciona que "la matemáticas en ingeniería es un lenguaje, puesto que casi todo lo que se dice en ingeniería se puede representar en lenguaje matemático" (p. 5), y que la modelación es uno de los temas que aparece en el currículo oculto, pero los profesores de matemáticas asumen que esto compete sólo a profesores de cursos propios de ingeniería; en contraste (Santos , 2008), manifiesta que deben seleccionarse apropiadamente problemas o actividades que promuevan el desarrollo del pensamiento matemático en los estudiantes, que le ayuden a construir una matemática para la vida, como herramienta de apoyo y disciplina formativa con una función específica en el nivel universitario donde los conocimientos deben ser integrados.

Además, la matemática es una de las ciencias básicas fundamentales en ingeniería, en cualquiera de sus especialidades; según (Cruz , 2010), hay dos razones fundamentales: por una parte se encuentra el conjunto de competencias intelectuales que se desarrollan al estudiar contenidos matemáticos (observación, análisis, comparación, síntesis, etc. y b) el hecho de constituir un poderoso lenguaje de comunicación de conocimientos de otras ramas que se usan en la formulación de situaciones ligadas a los llamados problemas de ingeniería (física, química, mecánica, electricidad, etc.), (p. 39). 
Por la trascendencia que tiene esta etapa en los procesos de modelación, la investigación se centra en éste elemento fundamental, analizando la estructura del lenguaje natural que requiere ser matematizado, $y$ en el marco del planteamiento de (Mayer , 1986), acerca de los estadios para resolver problemas, se planteó el siguiente objetivo para orientar la investigación: Determinar el tipo de significado que se establece en el conocimiento semántico cuando formulan ecuaciones diferenciales lineales de segundo orden como modelos matemáticos.

\section{Metodología}

La investigación fue cuantitativa de tipo exploratorio descriptivo. La población estuvo conformada por estudiantes que cursaron Ecuaciones Diferenciales en los programas de la Facultad Ingeniería de la Universidad Francisco de Paula Santander durante el II semestre académico 2016. La metodología que más se ajusta para la realización del trabajo es de tipo no experimental transeccional o transversal descriptivo, según Para la selección de los participantes se utilizó muestreo probabilístico (Hernández, Fernández, \& Baptista, 2006), "los diseños de investigación transeccional o transversal recolectan datos en un solo momento, en tiempo único; su propósito es describir variables, y analizar su incidencia e interrelación en un momento dado" (p. 187)

\section{Población y Muestra}

Se utilizó muestreo probabilístico para seleccionar 133 participantes de una población de 204 estudiantes de los programas de la facultad de ingeniería de la UFPS que cursaron la asignatura ecuaciones diferenciales durante el II semestre académico de 2016. Calculada a partir de la relación para poblaciones finitas:

$n=n o /\left(1+n_{0} / N\right), \quad$ allí $\quad n o=q(1-p)(Z(1-(\alpha / 2)) / d)^{2}$

donde: n: tamaño de la muestra

N: tamaño de la población

$Z$ : Valor correspondiente al nivel de confianza del 95\%

p: prevalencia esperada del parámetro a evaluar $\boldsymbol{q}=(1-p)$

\section{Instrumentos para recolección de información}

Para recolectar la información se diseñó un cuestionario de 17 reactivos con respuesta cerrada y abierta a partir de un problema de palabra que involucra contenidos del ciclo básico de ingeniería, donde el resolutor tiene que representar ecuaciones diferenciales ordinarias de segundo orden como modelos matemático. El diseño del cuestionario tuvo como fundamento conceptual tres ejes: la metodología de dos etapas para la resolución de problemas matemáticos propuesta por Mayer (1986), quien considera que el resolutor debe transitar por dos estadios: traducción y solución. Para el primero el solucionador debe tener conocimiento lingüístico, semántico y esquemático, y para el segundo conocimiento operativo y estratégico.
En lo que respecta a la modelación se orientó bajo la perspectiva cognitiva (Ferri , 2006). Allí la situación real se presenta mediante un problema, pude ser una imagen, un texto o los dos. La teoría de las representaciones internas (posibles configuraciones mentales de los individuos como solucionadores de problemas; no observables por supuesto) y externas (configuraciones observables tales como textos, gráficos, imágenes, ecuaciones o micro mundos informáticos, accesibles a la observación de cualquier persona con conocimientos adecuados), según (Goldin \& Kaput, 1996).

El siguiente problema de palabra fue tomado del texto Ecuaciones Diferenciales y problemas con valores en la frontera de ((Nagle , Saff, \& Snider, 2001), teniendo en cuenta que se realizaron algunas modificaciones, sirviendo como base conceptual para la aplicación del cuestionario.

\section{Problema Ejemplo:}

Considere un resorte de longitud 1 sujetado con firmeza a un punto fijo (como el techo). Una masa que pesa 16 libras se fija en el extremo inferior del resorte y lo alarga pie. La masa se libera inicialmente desde un punto 2 pies debajo de la posición de equilibrio y el movimiento posterior ocurre en un medio que ofrece una fuerza de amortiguamiento igual a de la velocidad instantánea. Formule la ecuación diferencial que rige el movimiento del sistema masa resorte si se aplica una fuerza externa igual a:

\section{$F(t)=10 \cos 3 t$}

\section{Validez y confiabilidad del instrumento}

En lo que hace referencia a la confiablidad por ser un estudio descriptivo y exploratorio, cuyos objetivos se centran en analizar la pluralidad de significados en el lenguaje natural (lengua castellana) que asocian a las palabras en las que aparece redactado el problema y los significados que otorgados a hechos del mundo real, signos, símbolos y expresiones matemáticas implicadas en el problema se esperaba que el estudiante responda con la mayor sinceridad posible las preguntas.

Respecto a la validez se dé constructo fue realizada por tres docentes con estudios de maestría en Educación Matemática. Como lo afirma (Martínez, 2009), "la validez de construcciones hipotéticas (de 'constructos') que es la más importante, trata de establecer una medida operacional para los conceptos usados" (p. 5).

\section{Resultados}

En este capítulo se muestran los hallazgos a partir a partir del análisis sociodemográfico, y el objetivo que orientó la investigación. 


\section{Análisis sociodemográfico}

La muestra estuvo conformada por 133 participantes de los cuales el $45,1 \%$ son mujeres. El $76 \%$ obtuvo su grado de bachiller en los años 2013 y 2014. En la Tabla 1 se puede observar que las edades de los estudiantes oscilan entre $17 \mathrm{y}$ 30 años, con un promedio de $19,44 \%$ años. El $80.4 \%$ tiene edades entre 18 y 20 años.

Tabla 1. Participantes por Edad

\begin{tabular}{|c|c|c|c|c|c|}
\hline & & Frecuencia & Porcentaje & $\begin{array}{c}\text { Porcentaje } \\
\text { válido }\end{array}$ & $\begin{array}{l}\text { Porcentaje } \\
\text { acumulado }\end{array}$ \\
\hline \multirow{11}{*}{ Válidos } & 17 & 3 & 2,3 & 2,3 & 2,3 \\
\hline & 18 & 43 & 32,3 & 32,3 & 34,6 \\
\hline & 19 & 38 & 28,6 & 28,6 & 63,2 \\
\hline & 20 & 26 & 19,5 & 19,5 & 82,7 \\
\hline & 21 & 9 & 6,8 & 6,8 & 89,5 \\
\hline & 22 & 7 & 5,3 & 5,3 & 94,7 \\
\hline & 23 & 4 & 3,0 & 3,0 & 97,7 \\
\hline & 26 & 1 & 0,8 & 0,8 & 98,5 \\
\hline & 28 & 1 & 0,8 & 0,8 & 99,2 \\
\hline & 30 & 1 & 0,8 & 0,8 & 100,0 \\
\hline & Total & 133 & 100,0 & 100,0 & \\
\hline
\end{tabular}

La Tabla 2 muestra la distribución de los participantes de acuerdo al programa de ingeniería que cursan. Se destaca que la mitad de ellos cursan programas de ingeniería Industrial y Civil, esto debido la ponderación realizada en el muestreo.

Tabla 2. Participantes por programa

\begin{tabular}{|c|c|c|c|c|c|}
\hline & & Frecuencia & Porcentaje & $\begin{array}{c}\text { Porcentaje } \\
\text { válido }\end{array}$ & $\begin{array}{l}\text { Porcentaje } \\
\text { acumulado }\end{array}$ \\
\hline \multirow{9}{*}{ Válidos } & $\begin{array}{l}\text { Ingenieriá } \\
\text { Industrial }\end{array}$ & 36 & 27,1 & 27,1 & 27,1 \\
\hline & $\begin{array}{c}\text { Ingenieria } \\
\text { Civil }\end{array}$ & 31 & 23,3 & 23,3 & 50,4 \\
\hline & $\begin{array}{l}\text { Ingenieria } \\
\text { Mecánica }\end{array}$ & 8 & 6,0 & 6,0 & 56,4 \\
\hline & $\begin{array}{l}\text { Ingeniería } \\
\text { de Minas }\end{array}$ & 22 & 16,5 & 16,5 & 72,9 \\
\hline & $\begin{array}{l}\text { Ingenieria } \\
\text { Electrónica }\end{array}$ & 12 & 9,0 & 9,0 & 82,0 \\
\hline & $\begin{array}{c}\text { Ingeniería } \\
\text { Electromecánica }\end{array}$ & 4 & 3,0 & 3,0 & 85,0 \\
\hline & $\begin{array}{l}\text { Ingeniería } \\
\text { de Sistemas }\end{array}$ & 18 & 13,5 & 13,5 & 98,5 \\
\hline & otro & 2 & 1,5 & 1,5 & 100,0 \\
\hline & Total & 133 & 100,0 & 100,0 & 99,2 \\
\hline
\end{tabular}

En la Tabla 3 se observa que los promedios académicos tienen un rango entre 2,91 y 4,48 en una escala numérica de 0 a 5 que tiene establecida y reglamentada la universidad.

\begin{tabular}{lcccccc}
\multicolumn{7}{c}{ Tabla 3. Promedio académico } \\
\hline & $N$ & Mínimo & Máximo & Media & $\begin{array}{c}\text { Desv. } \\
\text { típ. }\end{array}$ & Varianza \\
\hline $\begin{array}{c}\text { Promedio } \\
\text { académico } \\
\begin{array}{c}\text { Núlido } \\
\text { (según lista) }\end{array}\end{array}$ & 133 & 2,91 & 4,48 & 3,6053 &, 30180 &, 091 \\
\hline
\end{tabular}

La Tabla 4 muestra los resultados con sus respectivas ponderaciones. Allí se evidencia una gran variedad de representaciones externas que hace cada uno de los participantes a expresiones como: peso, masa, sistema masa resorte y ley de Hooke entre otros conceptos relacionados con la física.

Se observan también las diferentes formas de interpretar expresiones como: punto de equilibrio, fuerza amortiguadora, constante de elasticidad, proporcionalidad, velocidad como razón de cambio entre otros. Conceptos que tienen que ser evocados por el estudiante, ya que debieron ser abordados en los curos precedentes.

Tabla 4. Resultados externas a proposiciones y conceptos del instrumento de investigación

\begin{tabular}{|c|c|c|}
\hline Expresiones matemáticas & $\%$ & Representación Externa \\
\hline \multirow{4}{*}{$\begin{array}{c}\text { Un sistema masa resorte } \\
\text { está compuesto por }\end{array}$} & 30 & $\begin{array}{l}\text { Una masa puntual, un resorte ideal colgante y un } \\
\text { punto de sujeción del resorte, por ejemplo el techo }\end{array}$ \\
\hline & 10 & $\begin{array}{l}\text { Una masa } m \text { unida a un resorte ideal, que a la } \\
\text { vez se halla unido a una pared }\end{array}$ \\
\hline & 50 & $\begin{array}{l}\text { Un resorte con alto coeficiente de elasticidad, una } \\
\text { masa puntual y un punto de sujeción del resorte }\end{array}$ \\
\hline & 10 & $\begin{array}{l}\text { Un resorte con alto coeficiente de elasticidad que } \\
\text { no se deforme en su rango de estiramiento, una } \\
\text { masa puntual y un punto de sujeción del resorte }\end{array}$ \\
\hline \multirow{2}{*}{ La masa de un cuerpo es: } & 80 & $\begin{array}{l}\text { Una medida de la cantidad de materia que posee } \\
\text { un cuerpo y su unidad en el sistema internacional } \\
\text { de unidades es el kilogramo }\end{array}$ \\
\hline & 20 & $\begin{array}{l}\text { La inercia o resistencia a los cambios de estado de } \\
\text { movimiento }\end{array}$ \\
\hline \multirow{2}{*}{$\begin{array}{c}\text { El peso de un cuerpo, } \\
\text { representa }\end{array}$} & 90 & $\begin{array}{l}\text { El producto de su masa por la aceleración de la } \\
\text { gravedad, expresado mediante la relación } \\
\qquad \mathrm{W}=\mathrm{mg}\end{array}$ \\
\hline & 10 & La Fuerza con la que tierra atrae al cuerpo \\
\hline \multirow[t]{2}{*}{$\begin{array}{c}\text { La constante de elasticidad } \\
\text { del resorte, es }\end{array}$} & 90 & $\begin{array}{l}\text { El valor de la constante de proporcionalidad según } \\
\text { la ley de elasticidad de Hooke que establece: } \\
\text { dentro de los límites el estiramiento que } \\
\text { experimenta un material elástico es directamente } \\
\text { proporcional a la fuerza aplicada sobre el mismo }\end{array}$ \\
\hline & 10 & La Fuerza con la que tierra atrae al cuerpo \\
\hline \multirow{4}{*}{$\begin{array}{l}\text { El pie es la unidad de } \\
\text { longitud en el sistema }\end{array}$} & 40 & $\begin{array}{l}\text { De ingeniería y equivale a } 30.48 \mathrm{~cm} \\
\text { en el sistema Cgs }\end{array}$ \\
\hline & 20 & $\begin{array}{l}\text { De ingeniería y equivale a } 0.3048 \mathrm{~m} \\
\text { en el sistema Mks }\end{array}$ \\
\hline & 30 & $\begin{array}{l}\text { De ingeniería equivalente a } 30.48 \mathrm{~cm} \\
\text { en el sistema MKs. }\end{array}$ \\
\hline & 10 & $\begin{array}{l}\text { Cgs y equivale a } 12 \text { pulgadas } \\
\text { en el sistema de ingeniería }\end{array}$ \\
\hline \multirow{4}{*}{$\begin{array}{c}\text { La expresión: La masa se } \\
\text { libera inicialmente desde } \\
\text { un punto } 2 \text { pies debajo de } \\
\text { la posición de equilibrio, } \\
\text { significa: }\end{array}$} & 20 & $\begin{array}{l}\text { El momento a partir del cual se considera el } \\
\text { movimiento, es decir cuando } t=0\end{array}$ \\
\hline & 30 & $\begin{array}{c}\text { Representa una condición inicial expresada } \\
\text { mediante } \mathrm{y}(0)=2 \text {, allí } \mathrm{y}(\mathrm{t}) \text { es la función } \\
\text { desplazamiento del cuerpo }\end{array}$ \\
\hline & 40 & $\begin{array}{c}\text { Como el cuerpo es liberado debajo de la posición } \\
\text { de equilibrio la velocidad inicial es igual a cero y } \\
\text { se representa mediante dy/dt }=0\end{array}$ \\
\hline & 10 & $\begin{array}{c}\text { Las condiciones iniciales } \mathrm{y}(0)=2, \mathrm{y}^{\wedge \prime}(0)=0 \text { a partir } \\
\text { de las cuales se considera la situación y permiten } \\
\text { hallar la solución particular de la ecuación } \\
\text { diferencial }\end{array}$ \\
\hline \multirow{4}{*}{$\begin{array}{c}\text { Se puede afirmar que la } \\
\text { fuerza de amortiguamien- } \\
\text { to:La expresión: La masa } \\
\text { se libera inicialmente } \\
\text { desde un punto } 2 \text { pies } \\
\text { debajo de la posición de } \\
\text { equilibrio, significa: }\end{array}$} & 40 & $\begin{array}{l}\text { Tiene dirección opuesta al movimiento instantáneo } \\
\text { y es proporcional a la velocidad del cuerpo cuando } \\
\text { la velocidad es baja. }\end{array}$ \\
\hline & 10 & $\begin{array}{l}\text { Como es proporcional al movimiento del cuerpo, } \\
\text { existe la constante de amortiguamiento, } \\
\text { representada generalmente por la letra } c\end{array}$ \\
\hline & 30 & $\begin{array}{l}\text { Si dy/dt es positiva el cuerpo se mueve hacia abajo } \\
\text { y la fuerza de amortiguamiento se dirige hacia } \\
\text { arriba, representada mediante } \mathrm{F} \text { amost }=-c d y / d t \text {. }\end{array}$ \\
\hline & 20 & $\begin{array}{l}\text { Si dy/dt es negativa el cuerpo se mueve hacia } \\
\text { arriba y la fuerza de amortiguamiento se dirige } \\
\text { hacia abajo, representada mediante Famort }=-c d y d t .\end{array}$ \\
\hline
\end{tabular}




\section{Metodología}

Los hallazgos coinciden y muestran claramente que cada participante hace su propia representación interna y externa a conceptos como: sistema masa-resorte, peso, masa, punto de equilibrio, Ley de Hooke, fuerzo amortiguadora, fuerza externa, Ley de Newton, posiblemente estas se deben a su experiencia y competencias matemáticas coincidiendo con (Barbosa , 2006).

Los resultados evidencian también dificultades en el tránsito del lenguaje natural al lenguaje matemático y la representación externa de cada una de los signos, símbolos o expresiones matemáticas inmersas en el problema de palabra, como lo afirman: ((Haghverdi, Semnani, \& Seifi, 2012), (Calle Palomeque, 2013 ), (Bassanezi \& Biembengut, 1997 ), (Sabbagh , 2008) y (Berdugo , 2004).

La importancia de estudios relacionados con esta temática proviene desde hace cerca de 60 años, aunque esta tendencia sólo cobró fuerza en la década de 1980 (Suydam, 1980), citado en (Muir, Beswick, \& Williamson, 2008 ), por su parte el National Council of Teacher of Mathematics (Mathematics, 2000), afirmó que la resolución de problemas debería ser el foco central del currículo de matemáticas, entretanto Polya (1949) y otros como Branca (1980) citados por (Muir, Beswick, \& Williamson, 2008 ), sostienen que la resolución de problemas debe ser la meta de aprendizaje de las matemáticas, Gravemeijer y Doorma (1999), Zawojewski (2007) citados por (Santos, 2008 ), manifiestan que se deben "considerar situaciones auténticas o realistas que propicien en los estudiantes la construcción de modelos matemáticos" (p. 3), para Santos $(2008$, p. 3) existe "un gran número de propuestas curriculares en el ámbito internacional, que identifican la resolución de problemas como eje central de la organización de contenidos"; sin embargo hay poca evidencia, para afirmar que este enfoque está ocurriendo en las aulas de hoy (Anderson, 2003, Lovitt, 2000 y Schoenfeld, 1992 ), citados por (Muir, Beswick, \& Williamson, 2008 ).

\section{Conclusiones}

El trabajo evidencia la importancia que tiene la fase de representación en el ciclo de modelación y resolución de problemas. Cada uno de los participantes hace su propia representación externa a los diversos signos, símbolos y expresiones matemáticas involucradas en la situación, mostrando la importancia que tiene la fase de representación en el ciclo de modelación y resolución de problemas.

Debido al tránsito que se tiene que hacer del lenguaje en que se presenta el problema de palabra al lenguaje matemático construyendo una representación mental de la situación plasmada en una ecuación diferencial lineal de segundo orden con coeficientes constantes como modelo matemático.
Se evidencian también muestran dificultades en estas tareas; por tanto, es necesario realizar trabajos profundizando en el tema con el propósito de buscar explicaciones y contribuir en la enseñanza y aprendizaje de la matemática.

\section{Referencias}

Barbosa, J. (2006). Mathematical modelling in classroom: a socio - critical and discursive perspective. ZDM, 38, 39, 293 - 301.

Bassanezi, R., \& Biembengut, M. (1997). Modelación matemática: Una antigua forma de investigación un nuevo método de enseñanza. Números. Revista de didáctica de las matemáticas, 32, , 13-25.

Berdugo, O. (2004). Comprehension and representation of algebra word problems in a second language. Obtenido de Available from ProQuest Dissertations \& Theses A\&I: Social Sciences. (305064402).: http://search.proquest.com/docview/ 305064402 ?accountid $=43636$.

Blomhøj, M. (2008). Different perspectives on mathematical modelling in educational research-Categorising the TSG21 papers. In ICME 11 international Congress on Mathematics Education, 1-13.

Blum, W., \& Ferri, R. (2009). Mathematical modelling: Can it be taught and learnt? ournal of mathematical modelling and application, 1(1), 45-48.

Calle Palomeque, C. (2013). Influencia de la semántica en el segundo curso de bachillerato del colegio Benigno Malo. Obtenido de Tesis de Maestsría: http://dspace.ucuenca.edu.ec/handle/123456789/4693 .

Camarena, G. P. (2014 de Diciembre de 2004). La matemática en el contexto de las ciencias. Obtenido de Repositorio Digital de documentos en Educación Matemática - FUNES: http://funes.uniandes.edu.co/6234/.

Camarena, G. P. (2012). La modelación matemática en la formación del ingeniero. Revista Brasilera de Ensino de Ciencia y Tecnología. 5(3), 1-10.

Cruz, C. (2010). La enseñanza de la modelación matemática en ingeniería. Revista de la Facultad de Ingeniería de Universidad Central de Venezuela, 39-46.

Ferri, R. (2006). Theoretical and empirical differentiations of phases in the modelling process. ZDM, 38(2), 86-95.

Gallardo, P. (s.f.). La modelación matemática en el ambiente de aprendizaje: una innovación. Obtenido de Premio Nacional ANUIES 2000 a la mejor tesis de doctorado en contribución a la Eduación Superior: http://www.repo-ciie.cgfie.ipn.mx/ pdf/625.pdf. 
Goldin, G., \& Kaput, J. (1996). A joint perspective on the idea of representation in learning and doing mathematics. . Theories of mathematical learning, 397-430.

Gutiérrez, J. (2014). Las matemáticas en Ingeniería: todo un reto pedagógico. Innovación, Ingeniería y Desarrollo, 1(1), 75-80.

Haghverdi, M., Semnani, A., \& Seifi, M. (2012). The relationship between different kinds of students' errors and the knowledge required to solve mathematics word problems. Bolema: Boletim de Educação Matemática, 26(42B), 649-666.

Hernández, R., Fernández, C., \& Baptista, P. (2006).Metodología de la Investigación. Cuarta Edición. México: McGraw-Hill .

Martínez, M. (2009). Nuevos paradigmas en la investigación. Venezuela: Alfa .

Mathematics, N. C. (2000). Estándares Curriculares y de evaluación para la educación matemática. Obtenido de http://www.nctm.org/uploadedFiles/Standards_and_Positions/PSSM_ExecutiveSummary.pdf

Mayer, R. (1986). Thinking, Problem Solving, Cognition. Barcelona: (Trad. Graziella Baravella). (1a ed.). Barcelona: Ediciones Paidos. (Original publicado en 1983).

Mayer, R., Lewis, A., \& Hegarty, M. (1992). Mathematical misunderstandings: Qualitative reasoning about quantitative problems. Advances in psychology, 91, 137-153.

Muir, T., Beswick, K., \& Williamson, J. (2008). "I'm not very good at solving problems": An exploration of students' problem solving behaviours. The Journal of Mathematical Behavior, 27(3), 228-241.

Nagle, R., Saff, E., \& Snider, A. (2001). Ecuaciones diferenciales y problemas con valores en la frontera. Pearson Educación .

Polya, G. (2005). Polya, G. (2005). Cómo plantear y resolver problemas. (reimp. XVII). . Mexico: (Trad. Zagazagoitia) Mexico: Editorial Trillas. (Original publicado en 1965).

Sabbagh, S. (2008). Solución de problemas aritméticos redactados y control inhibitorio cognitivo. Universitas Psychologica, 7 (1), 217-229.

Santos, L. (07 de Marzo de 2008). La Resolución de problemas: Avances y perspectivas en la construcción de una agenda de investigación y práctica. Obtenido de Memorias de seminario de Resolucón de Problemas: 30 años después del XII Simposio de la Sociedad Española de Investigación en Educación Matemática:http://www.uv.es/puigl/MSantosTSEIEM08.pdf

Shonenfeld, A. (1980). Enseñanza de habilidades para resolver problemas. he American Mathematical Monthly , 87 (10), 794-805.
Wright, J. (2014). An investigation of factors affecting student performance in algebraic word problem solutions. Obtenido de Available from ProQuest Dissertations \& ThesesA\&I: http://search.proquest.com/docview/1630101245?accounti$\mathrm{d}=43636$. 